\title{
Immunostimulatory effect of faecal Bifidobacterium species of breast-fed and formula-fed infants in a peripheral blood mononuclear cell/Caco-2 co-culture system
}

\author{
T. Pozo-Rubio ${ }^{1}$, J. R. Mujico ${ }^{1}$, A. Marcos $^{1}$, E. Puertollano ${ }^{2}$, I. Nadal ${ }^{3}$, Y. Sanz ${ }^{3}$ and E. Nova* \\ ${ }^{1}$ Immunonutrition Group, Metabolism and Nutrition Department, Instituto de Ciencia y Tenología de Alimentos y Nutrición \\ (ICTAN-CSIC), C/Jose Antonio Novais, 10, 28040 Madrid, Spain \\ ${ }^{2}$ Division of Microbiology, Department of Health Sciences, Faculty of Experimental Sciences, University of Jaén, Jaén, Spain \\ ${ }^{3}$ Microbial Ecophysiology and Nutrition Group, Instituto de Agroquímica y Tecnología de Alimentos (CSIC), Valencia, Spain \\ (Received 24 September 2010 - Revised 19 January 2011 - Accepted 28 February 2011 - First published online 31 May 2011 )
}

\section{Abstract}

Bifidobacterium spp. typical of the human intestinal microbiota are believed to influence the balance of immune responses in the intestinal mucosa. The aim of the present study was to investigate the effect of different bifidobacterial species and their mixtures in in vitro experiments with peripheral blood mononuclear cells (PBMC) and Caco-2 cells. Bifidobacterium adolescentis, B. angulatum, B. breve, B. catenulatum, B. infantis, B. longum and two combinations of these bifidobacteria simulating the species composition found in faecal samples from breast-fed (BF) and formula-fed (FF) infants were used. The levels of several cytokines were measured by direct stimulation of PBMC and by stimulation of a Caco-2/PBMC co-culture with bifidobacteria. B. catenulatum and $B$. breve were the strongest enhancers of interferon- $\gamma$ (IFN- $\gamma$ ) production by direct stimulation of PBMC. B. longum was the highest inducer of IL-10 and the lowest TNF- $\alpha$ stimulus. In the Caco-2/PBMC system, $B$. breve was the highest inducer of IL- 8 production by Caco- 2 cells, significantly different from B. infantis, $B$. adolescentis and the FF mixture $(P<0 \cdot 05)$. IFN- $\gamma$ produced by PBMC stimulated with the BF mixture (containing $22 \% B$. breve, compared with $7 \%$ in the FF mixture) was significantly higher compared with B. adolescentis, B. infantis and B. longum. B. adolescentis also inhibited IFN- $\gamma$ production compared with the FF mixture and B. longum. The proportion of different Bifidobacterium strains seems to be an important determinant of the cytokine balance in the simulated intestinal environment studied. $B$. breve and the combination of the Bifidobacterium species typically found in the microbiota of BF infants have shown the most significant effects.

\section{Key words: Bifidobacterium spp.: Caco-2 cells: Peripheral blood mononuclear cells: Cytokines: Breast-feeding: Formula} feeding: Infant's microbiota

The intestinal microbiota plays a pivotal role in human health by preventing pathogen colonisation, and shaping and maintaining normal mucosal immunity ${ }^{(1)}$. To preserve this beneficial relationship, the immune system should remain hyporesponsive to commensal bacteria (mucosal tolerance $)^{(2,3)}$, but at the same time, it has to combat pathogenic bacteria $^{(3)}$. The breakdown of the delicate balance of the intestinal immune responses causes the development of disease states with bowel inflammation ${ }^{(3)}$. In this context, intestinal epithelial cells (IEC) play an important role in immune homeostasis ${ }^{(4,5)}$. IEC are thought to contribute to immunomodulation of mucosal leucocytes by at least two different mechanisms ${ }^{(6)}$, by acting as a physical barrier between gut luminal content (including bacteria) and the underlying immune cells, and by transmitting signals coming from the intestinal content and microbiota to the resident mucosal immune system ${ }^{(4)}$. IEC secrete many mediators involved in protective responses against potentially pathogenic organisms, such as defensins, mucins, chemokines and cytokines $^{(5)}$.

Bifidobacteria, which are important components of the human intestinal microbiota particularly of breast-fed (BF) infants $^{(7)}$, have shown the capacity to modulate cytokine production by IEC, monocyte-derived dendritic cells and peripheral blood mononuclear cells (PBMC) in in vitro experiments $^{(1,8,9)}$. In addition, the differences observed in the composition of bifidobacterial species of the intestinal microbiota of $\mathrm{BF}$ and formula-fed (FF) infants have been suggested to influence the incidence of immune-mediated diseases ${ }^{(10,11)}$. These findings have led to the proposal to

Abbreviations: BF, breast-fed; FF, formula-fed; IEC, intestinal epithelial cells; IFN- $\boldsymbol{\gamma}$, interferon- $\boldsymbol{\gamma}$; PBMC, peripheral blood mononuclear cells. 
use some Bifidobacterium strains as potential probiotics in the prevention and treatment of pathologies with underlying immune alterations, such as inflammatory bowel diseases, allergy and coeliac disease ${ }^{(12-14)}$.

Following all of the aforementioned facts and hypothesis, the objective of the present study was to investigate the effect of strains of different bifidobacterial species (Bifidobacterium adolescentis, B. angulatum, B. breve, B. catenulatum, B. infantis and B. longum) and their mixtures, corresponding to the typical microbiota present in the faeces from $\mathrm{BF}$ and $\mathrm{FF}$ children, on the modulation of cytokine production by IEC and PBMC in an in vitro co-culture system, simulating the intestinal environment.

\section{Materials and methods}

\section{Bacteria}

The following strains of six different Bifidobacterium species were individually evaluated: $B$. adolescentis ATCC 15703; B. angulatum ATCC 27535; B. breve ATCC 15700; B. catenulatum LMG 11043; B. longum biovar infantis LMG 11046T; B. longum biovar longum ATCC 15707. In addition, two combinations of these bifidobacteria were also used to simulate the percentage of each species in the microbiota from $\mathrm{BF}$ and $\mathrm{FF}$ infants ${ }^{(10)}$. The $\mathrm{BF}$ mixture included B. infantis $(59.0 \%), \quad B$. breve $(21.6 \%), B$. longum (13.5\%), B. catenulatum (3.5\%), B. angulatum (1.8\%) and $B$. adolescentis $(0.6 \%)$; the FF mixture included $B$. infantis (62.1\%), B. catenulatum (14.8\%), B. longum (10.9\%), $B$. breve $(7 \cdot 2 \%)$ and $B$. adolescentis $(5 \cdot 0 \%)$ (no B. angulatum).

Bifidobacteria were grown routinely in de Man Rogosa and Sharpe agar (Scharlau Chemie SA, Barcelona, Spain) with $0.05 \%$ cysteine broth and incubated at $37^{\circ} \mathrm{C}$ under anaerobic conditions (AnaeroGen, Oxoid, Basingstoke, UK) for $22 \mathrm{~h}$. Cells were harvested by centrifugation ( $6000 \boldsymbol{g}$ for $15 \mathrm{~min}$ ) until the stationary growth phase, washed two times in PBS (130 mm-NaCl, $10 \mathrm{~mm}$-sodium phosphate, $\mathrm{pH} 7 \cdot 4$, and resuspended in PBS containing $20 \%$ glycerol). Aliquots of these suspensions were frozen in liquid $\mathrm{N}_{2}$ and stored at $-80^{\circ} \mathrm{C}$ until used. The number of live cells after storage was determined by colony-forming unit counting on de Man Rogosa and Sharpe-cysteine after $48 \mathrm{~h}$ incubation in optimal conditions. For all strains tested, $>90 \%$ of cells were alive upon thawing. For every new experiment, one fresh aliquot was thawed to avoid variability in cultures between the experiments.

\section{Leucocyte isolation and bacterial stimulation of peripheral blood mononuclear cells}

Human PBMC from seven healthy volunteers were isolated from heparinised blood samples using standard Ficoll gradient centrifugation (lymphocyte isolation solution; Rafer, Zaragoza, Spain). The isolated PBMC were washed twice with Roswell Park Memorial Institute 1640 medium (Bio-Whittaker, Verviers, Belgium) and suspended in the same medium, supplemented with heat-inactivated fetal bovine serum $(100 \mathrm{ml} / \mathrm{l}$;
Bio-Whittaker), after decomplementation, and containing $1 \%$ penicillin-streptomycin $(5000 \mathrm{IU} / \mathrm{ml}(3 \mathrm{mg} / \mathrm{ml}), 5000 \mathrm{mg} / \mathrm{ml}$; Bio-Whittaker). The PBMC suspension was adjusted to $2 \times 10^{6}$ cells $/ \mathrm{ml}$, and $1 \times 10^{6}$ cells were used per well in all experiments.

Live bacterial cell suspensions of each individual Bifidobacterium strain or the combinations representing the faecal microbiota composition of $\mathrm{BF}$ and FF infants were washed in the culture medium and incubated at a final concentration of $10^{7}$ colony-forming units/ml with PBMC (proportion bacteria:PBMC, $10: 1)^{(15,16)}$ during $48 \mathrm{~h}\left(5 \% \mathrm{CO}_{2}\right.$ and $\left.37^{\circ} \mathrm{C}\right)$. The supernatant was collected, centrifuged and frozen in aliquots at $-80^{\circ} \mathrm{C}$ until cytokine analysis.

\section{Co-culture of Caco-2/peripheral blood mononuclear cells and bacterial stimulation}

The colonic adenocarcinoma cell line Caco-2 (ECACC no. 86010202 , Salisbury, Wiltshire, UK) was cultured at $37^{\circ} \mathrm{C}$ and $5 \% \mathrm{CO}_{2}$ in Eagle's minimal essential medium (Bio-Whittaker) supplemented with $10 \%$ fetal bovine serum (Bio-Whittaker), $1 \%$ non-essential amino acid solution (Bio-Whittaker), 1\% L-glutamine (Bio-Whittaker) and $1 \%$ penicillin-streptomycin (Bio-Whittaker). Caco-2 cells were seeded at a density of $8 \times 10^{4}$ cells/well in standard twentyfour-well culture plates, and at $4 \times 10^{4}$ cells/well on $12 \mathrm{~mm}$ inserts in twenty-four-well cell culture plate assemblies (Millipore, Madrid, Spain) with a semipermeable polyethylene terephthalate membrane ( $1 \mu \mathrm{m}$ in pore size). During cell growth and differentiation, the medium was changed every 2 or $3 \mathrm{~d}$. Once the cells were confluent and differentiated, the experiments were performed $10-11 \mathrm{~d}$ after seeding. Confluence was followed by microscopic visualisation and transepithelial resistance measurements (Millicell ERS Ohmmeter; Millipore).

Co-cultures of the bifidobacteria with Caco-2 cells and PBMC from healthy donors were performed in seven different experiments. To that end, a transwell cell culture system was used as described earlier. Caco- 2 monolayers were challenged by apical addition of $2 \times 10^{6}$ colony-forming units/insert of a Bifidobacterium strain or a combination of strains corresponding to the species composition in the faecal samples from BF and FF infants. The PBMC suspension $(500 \mu \mathrm{l})$ was added at a concentration of $2 \times 10^{6}$ cells $/ \mathrm{ml}$ in the basal compartment of the culture well for a $12 \mathrm{~h}$ incubation. Thereafter, further $36 \mathrm{~h}$ incubation was allowed after disassembly of the system. In order to measure the cytokine production by the sensitised Caco-2 and PBMC separately, the basolateral compartment of Caco-2 cells was replenished with a fresh culture medium. After the incubation period, culture media, from both the separated PBMC and Caco- 2 cell plates, were collected and frozen in aliquots at $-80^{\circ} \mathrm{C}$. The PBMC supernatant was centrifuged before freezing to avoid the presence of cells in aliquots.

In two different wells, two more conditions, which served as a control of the Caco- 2 cell conditioning by the underlying PBMC, were carried out: the BF and FF mixtures were added to the Caco-2 monolayers in transwells with no PBMC in the basal compartment. 


\section{Cytokine quantification in culture supernatants}

TNF- $\alpha$, IL-1 $\beta$, IL-10, IL-8 and IL-6 cytokines were measured in the basolateral medium with Caco- 2 cells, and TNF- $\alpha$, interferon- $\gamma$ (IFN- $\gamma$ ), IL-6, IL-10, IL-2 and IL-4 were measured in the PBMC supernatant. All cytokine measurements were performed using a cytometric bead array system (Inflammation Kit and either a Th1/Th2 kit or a Flex set; BD Biosciences, San Agustín de Guadalix, Madrid, Spain), according to the manufacturer's protocols, and analysed by flow cytometry (FACScalibur; BD Biosciences). Data were analysed using Cellquest software (BD Biosciences). The cytometric bead array limit of detection for each cytokine was as follows: IFN- $\gamma$, $7 \cdot 1 \mathrm{pg} / \mathrm{ml} ; \mathrm{TNF}-\alpha, 2 \cdot 8 \mathrm{pg} / \mathrm{ml}$; IL-10, $0 \cdot 13 \mathrm{pg} / \mathrm{ml} ; \mathrm{IL}-6,1 \cdot 6 \mathrm{pg} / \mathrm{ml}$; IL-8, $\quad 1.2 \mathrm{pg} / \mathrm{ml} ; \quad$ IL- $4, \quad 2.6 \mathrm{pg} / \mathrm{ml} ; \quad$ IL- $2, \quad 2.6 \mathrm{pg} / \mathrm{ml} ; \quad$ IL- $1 \beta$, $7 \cdot 2 \mathrm{pg} / \mathrm{ml}$. IFN- $\boldsymbol{\gamma}$ was also measured with high-sensitivity Immunoassay xMAP Technology (Millipore) in a Luminex 100 equipment, with a sensitivity of $0 \cdot 29 \mathrm{pg} / \mathrm{ml}$.

\section{Statistical analyses}

Statistical analyses were performed using SPSS version 17.0 software (SPSS, Inc., Chicago, IL, USA). To establish the homogeneity of variances and the distribution of the data, the Levene test was run. As a result of the non-normal distribution of the data and the non-homogeneity of the variances, the Mann-Whitney $U$ test was used to assess the effect of every experimental condition compared with the other conditions. Data are expressed as medians and quartiles. Significant differences were established at $P<0 \cdot 05$. Correlations between different bacterial stimulatory conditions were analysed by Spearman's correlation test and considered significant at a $P$ level $<0 \cdot 05$.

\section{Ethical approval}

The present study was conducted according to the guidelines laid down in the Declaration of Helsinki, and all procedures involving human subjects/patients were approved by the Ethics Committees of the Hospital Puerta de Hierro (Madrid, Spain) and CSIC organisation. Written informed consent was obtained from all subjects/patients.

\section{Results}

\section{Cytokine production by peripheral blood mononuclear cells cultured with bifidobacteria}

In order to determine the immunological effect of bifidobacteria on PBMC, the production of IFN- $\gamma$, TNF- $\alpha$, IL-10, IL-6, IL- 4 and IL-2 was measured in the supernatants of PBMC cultured in direct contact with the different Bifidobacterium strains (individually or mixed). Among all cytokines analysed, only IL-2 was not stimulated (Fig. 1(A)), with levels below $20 \mathrm{pg} / \mathrm{ml}$ (except for the positive control with phytohaemaglutinin; data not shown). All the other cytokines were significantly stimulated by all bifidobacterium species and their mixtures (compared with the control with only medium).

Regarding IFN- $\gamma$ production (Fig. 1(B)), B. catenulatum and $B$. breve were the strongest enhancers, followed by the FF and
BF mixtures (no significant differences were found between the two mixtures). B. catenulatum induced a higher IFN- $\gamma$ production than all the other stimuli (except for $B$. breve). $B$. breve induced a higher IFN- $\gamma$ production than $B$. adolescentis, $B$. angulatum and $B$. infantis, but similar to that induced by $B$. catenulatum, $B$. longum and the mixtures. The total percentage of $B$. catenulatum and $B$. breve was similar in the FF and BF mixture (22.05 and $25.12 \%$, respectively). This might explain why the levels of IFN- $\gamma$ produced by PBMC stimulated with the FF and BF mixtures were not statistically different.

B. longum and B. catenulatum induced the highest IL-10 production by PBMC, showing significant differences in IL-10 production in the presence of $B$. infantis and the BF mixture (Fig. 1(C)). IL-10 production induced by B. longum was also significantly higher than that induced by $B$. angulatum, $B$. breve and the FF mixture. The percentages of $B$. longum in the $\mathrm{FF}$ and $\mathrm{BF}$ mixtures were very similar (10.87 v. $13.52 \%)$, but B. catenulatum was approximately four times higher in the FF mixture than in the BF mixture $(14.84 \mathrm{v} .3 .50 \%)$. The low proportion of $B$. catenulatum and $B$. adolescentis, together with the high proportion of $B$. infantis and $B$. breve in the $\mathrm{BF}$ mixture, might explain the significantly lower production of IL-10 induced by the $\mathrm{BF}$ mixture compared with that induced by $B$. adolescentis, B. catenulatum and B. longum individually (Fig. 1(C)). Regarding IL-4, B. catenulatum also induced a significantly higher production than $B$. adolescentis and B. infantis (Fig. 1(D)).

All Bifidobacterium strains stimulated PBMC to produce very high levels of IL-6, over 4000 pg/ml (Fig. 1(E)). B. adolescentis induced the highest IL- 6 production, significantly higher than B. angulatum, B. breve and B. infantis $(P=0.029$ in every case). $B$. infantis induced the lowest effect among the assayed strains on cytokine production, not only for IL-6, but also for IFN- $\gamma$, IL-10 and IL-4.

With the exception of $B$. adolescentis, all Bifidobacterium strains also stimulated PBMC to produce very high levels of TNF- $\alpha$ (Fig. 1(F)). A significantly higher TNF- $\alpha$ production was induced by $B$. angulatum and $B$. catenulatum compared with $B$. adolescentis, $B$. breve and B. longum. While B. longum and $B$. adolescentis induced a high production of IL-10, they both mildly induced TNF- $\alpha$ production (Fig. 1(C) and (F)). On the other hand, while $B$. infantis and $B$. angulatum induced a mild production of IL-10, they both highly induced TNF- $\alpha$ production (Fig. 1(C) and (F)).

\section{Cytokine production by peripheral blood mononuclear cells in co-culture with Caco-2 cells and bifidobacteria}

To analyse cytokine production by PBMC conditioned by previous co-culture with Caco- 2 cells stimulated with bifidobacteria, IFN- $\gamma$, TNF- $\alpha$, IL-10, IL-6, IL-4 and IL-2 were measured in PBMC supernatants. IL-2 and IL- 4 were not detectable, and TNF- $\alpha$ was also below the limit or approaching the limit of detection (data not shown). No significant differences in IL-10 and IL-6 production were found, either between different bifidobacteria alone or in mixtures (Fig. 2(A) and (B)).

The production of IFN- $\gamma$ by PBMC was low in this system (range $1-93 \mathrm{pg} / \mathrm{ml}$ and under the detection limit in two of 
(A)

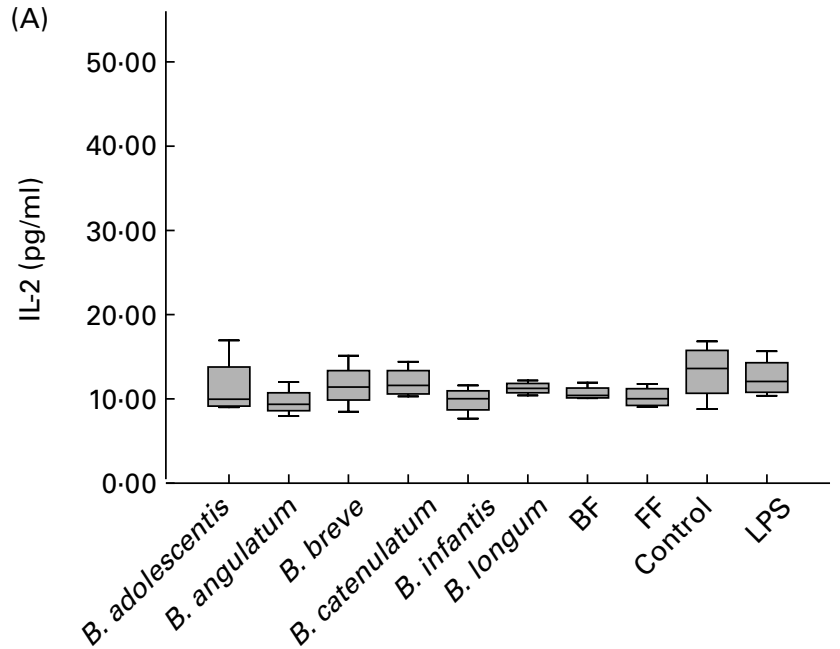

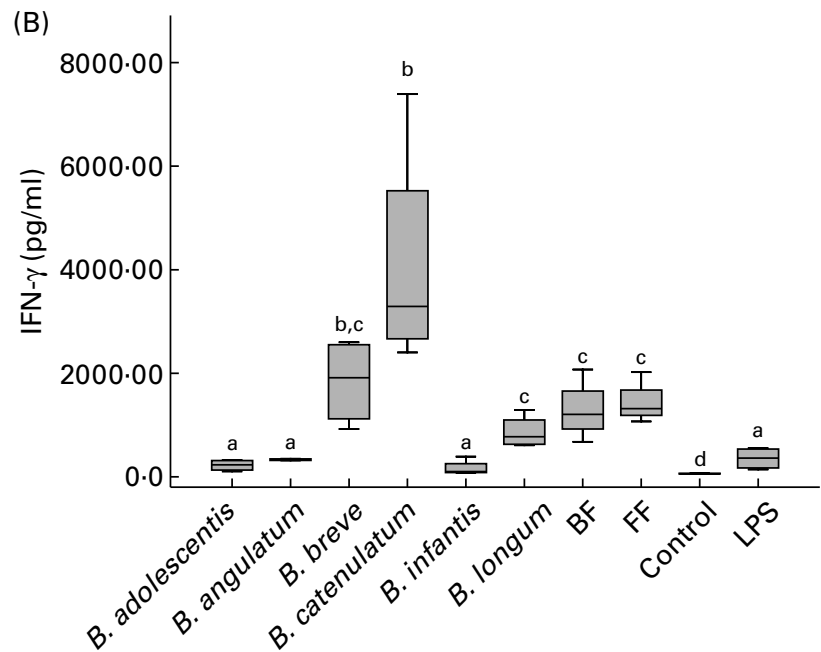

(C)

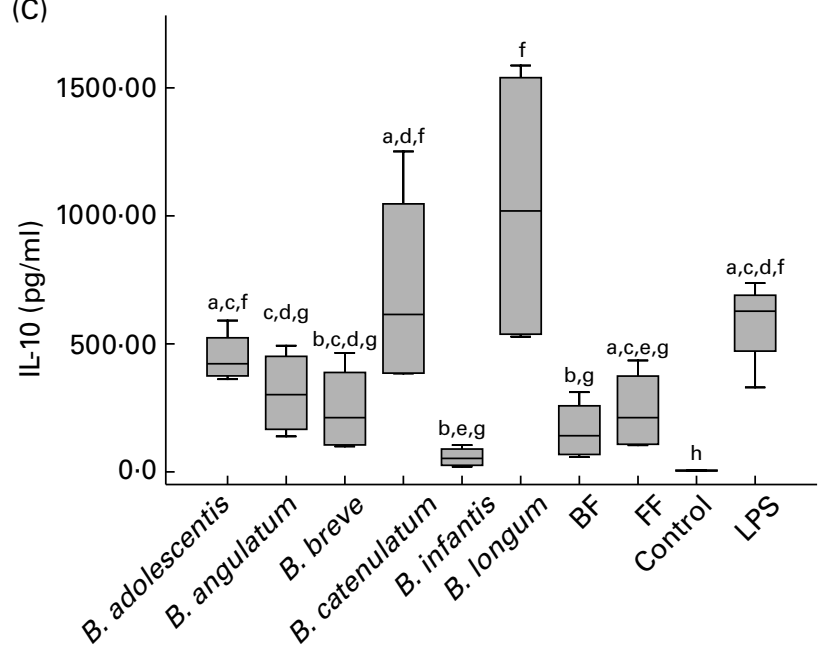

(D)

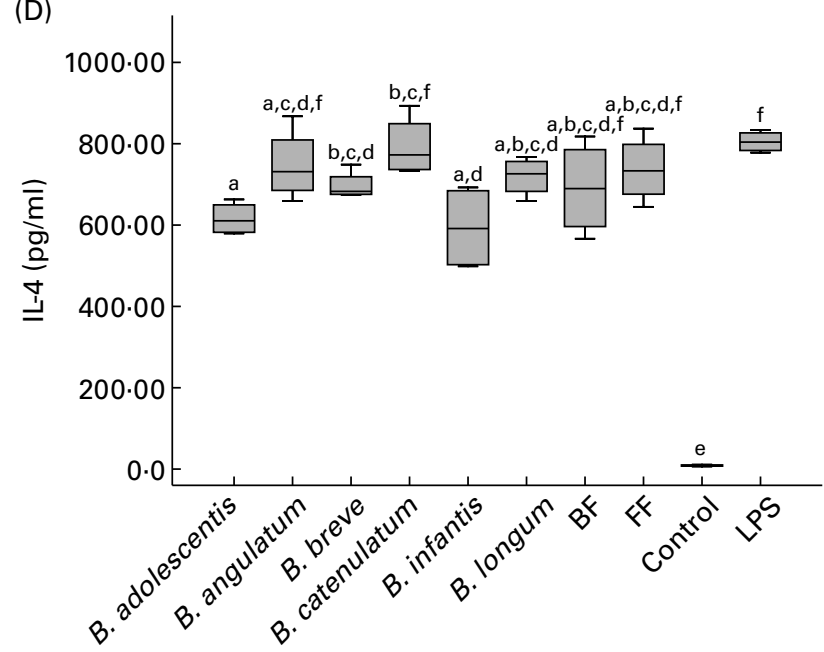

(E)

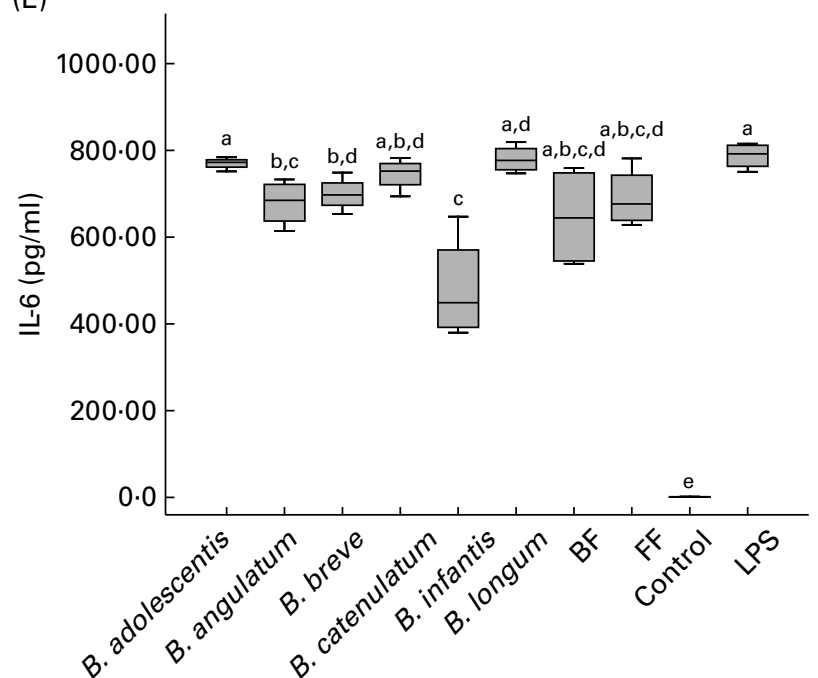

$(\mathrm{F})$

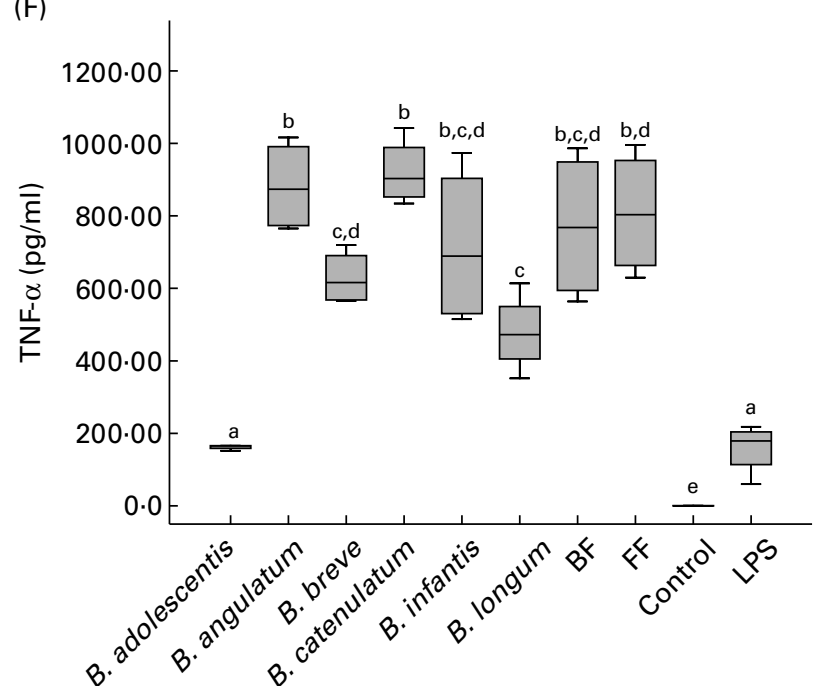

Fig. 1. Cytokine production by peripheral blood mononuclear cells after $48 \mathrm{~h}$ incubation with individual bifidobacterium strains and their mixtures (breast-fed (BF) and formula-fed (FF)) in a 10:1 (bacteria:cell) ratio. Each box represents median (50th percentile) and interquartile range (25th and 75 th percentiles). ${ }^{\text {a,b,c,d,e,f,g }}$ Mean values with unlike letters were significantly different $(P<0.05$; Mann-Whitney $U$ test). No differences were observed in IL-2 production between the conditions. (A) IL-2; (B) interferon- $\gamma$ (IFN- $\gamma$ ); (C) IL-10; (D) IL-4; (E) IL-6; (F) TNF- $\alpha$. LPS, lipopolysaccharide. 

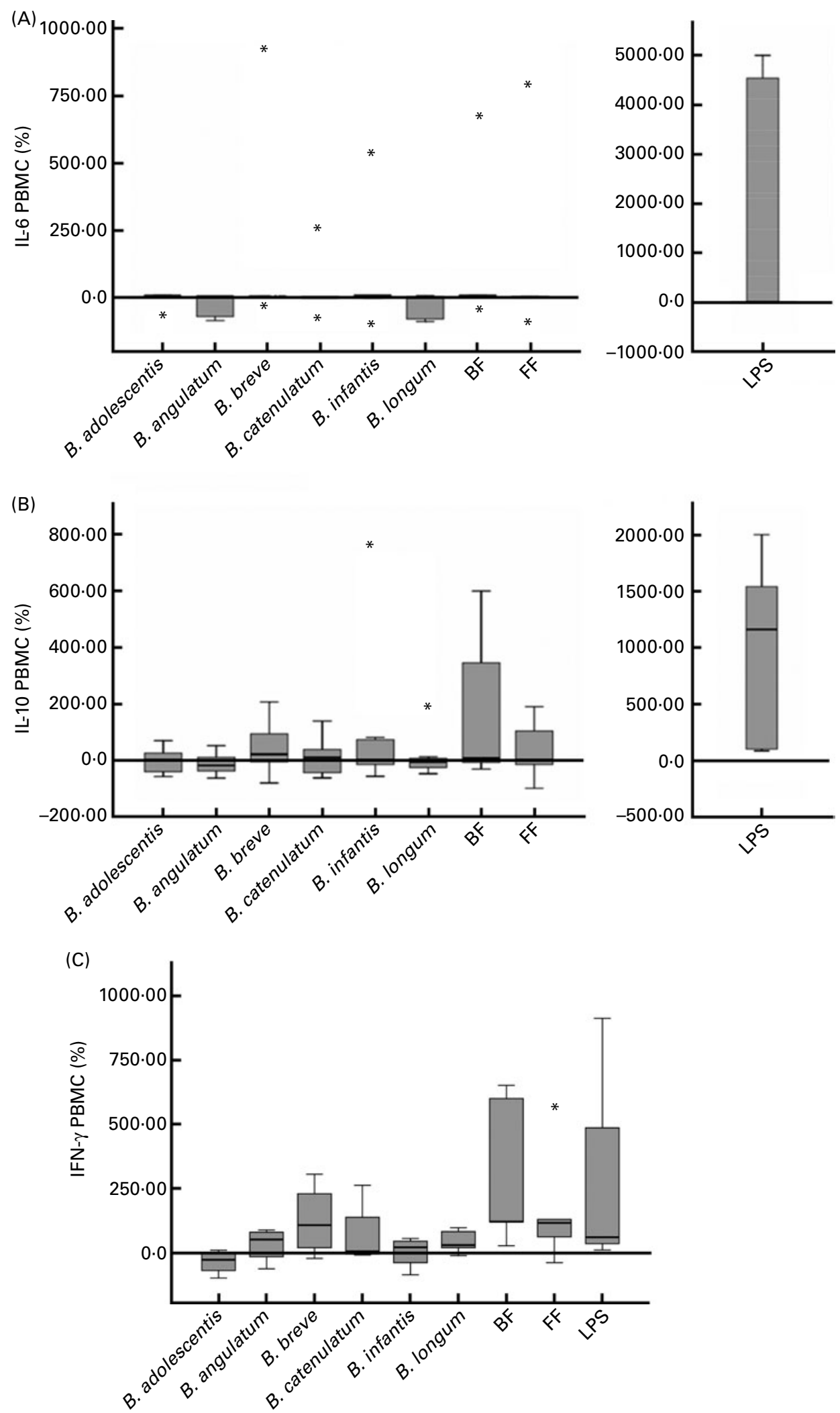

Fig. 2. Cytokine production in a $48 \mathrm{~h}$ culture of peripheral blood mononuclear cells (PBMC) sensitised by a $12 \mathrm{~h}$ incubation in a transwell co-culture system with Caco-2 cells apically stimulated with bifidobacteria. Values are given as percentage of the control (spontaneous production with no added bacteria). Each box represents median (50th percentile) and interquartile range (25th and 75th percentiles). Asterisks represent outliers. No differences were observed in IL-10 and IL-6 production between the different bifidobacterium conditions employed; however, lipopolysaccharide (LPS)-stimulated production was always significantly higher than the rest of the conditions. (A) IL-6 PBMC; (B) IL-10 PBMC; (C) interferon- $\gamma$ PBMC. BF, breast fed; FF, formula fed. 
the seven PBMC donors). Using the available data from the other five donors, we found induction of IFN- $\gamma$ production by the $\mathrm{BF}$ mixture in four of them $(>100 \% v$. control) and in three of them also with $B$. breve ( $>50 \% v$. control), which is singularly high in the BF combination. Moreover, three donors showed stimulation with the FF mixture ( $>100 \% v$. control). The BF mixture was the stimulus that induced the highest IFN- $\gamma$ production (Fig. 2(C)), significantly higher than $B$. adolescentis $(P=0 \cdot 014), B$. infantis $(P=0 \cdot 050)$ and $B$. longum $(P=0.047)$ individually. Although $B$. breve also induced the production of IFN- $\gamma$, this effect was not significantly different from the other bifidobacteria (Fig. 2(C)). The effect of $B$. adolescentis on IFN- $\gamma$ induction was inhibitory relative to the control condition and was significantly different from the stimulatory effect observed with the bifidobacteria mixtures and B. longum (Fig. 2(C)).

\section{Cytokine production by Caco-2 cells in co-culture with} peripheral blood mononuclear cells and bifidobacteria

To assess the effects of bifidobacteria and bifidobacteria mixture stimulation on Caco- 2 cells in co-culture with PBMC, TNF$\alpha$, IL-1 $\beta$, IL-10, IL-8 and IL- 6 cytokines were measured in both apical and basolateral media. All cytokines were not detectable in the apical medium, while in the basolateral medium only IL- 8 and IL- 6 were in a measurable concentration range (IL-8, 120-14000 pg/ml; IL-6, 30-600 pg/ml). When Caco-2 cells were stimulated with the bifidobacteria alone, with no PBMC in the underlying compartment, the stimulation of both cytokines was three to four times lower than in the co-culture system (data not shown).

When in co-culture with PBMC, $B$. breve highly stimulated the production of IL- 6 and IL- 8 on Caco- 2 cells ( 66.8 and $45.5 \%$, respectively; Fig. 3(A) and (B)). For IL- 8 , this production was significantly higher, compared with $B$. adolescentis $(P=0.035), B$. infantis $(P=0.025)$ and the FF mixture $(P=0.013)$ (Fig. 3(B)). Although the BF mixture also induced IL-6 and IL-8 production (36.0 and $20.7 \%$, respectively), these values were not significantly higher than those induced by the FF mixture (Fig. 3(A) and (B)). No significant differences were observed for IL-6 production between the different stimuli assayed (Fig. 3(A)).

Considering the PBMC donors individually, IL-8 and IL-6 production stimulated by the $\mathrm{FF}$ mixture was positively and significantly correlated with IL- 8 and IL-6 production stimulated by B. infantis ( $P<0.001$ for both cytokines). On the other hand, IL- 8 production stimulated by the BF mixture was correlated with $B$. angulatum, $B$. breve and $B$. catenulatum $(P<0.05)$, and IL-6 stimulated by the BF mixture correlated with $B$. adolescentis and B. catenulatum $(P<0 \cdot 05)$.

\section{Discussion}

Bifidobacterium strains have shown the capacity to modulate cytokine production by IEC, monocyte-derived dendritic cells and PBMC in in vitro experiments ${ }^{(1,8,9)}$. Trying to define this immunomodulatory capacity seems relevant in order to understand their contribution to the establishment of mucosal
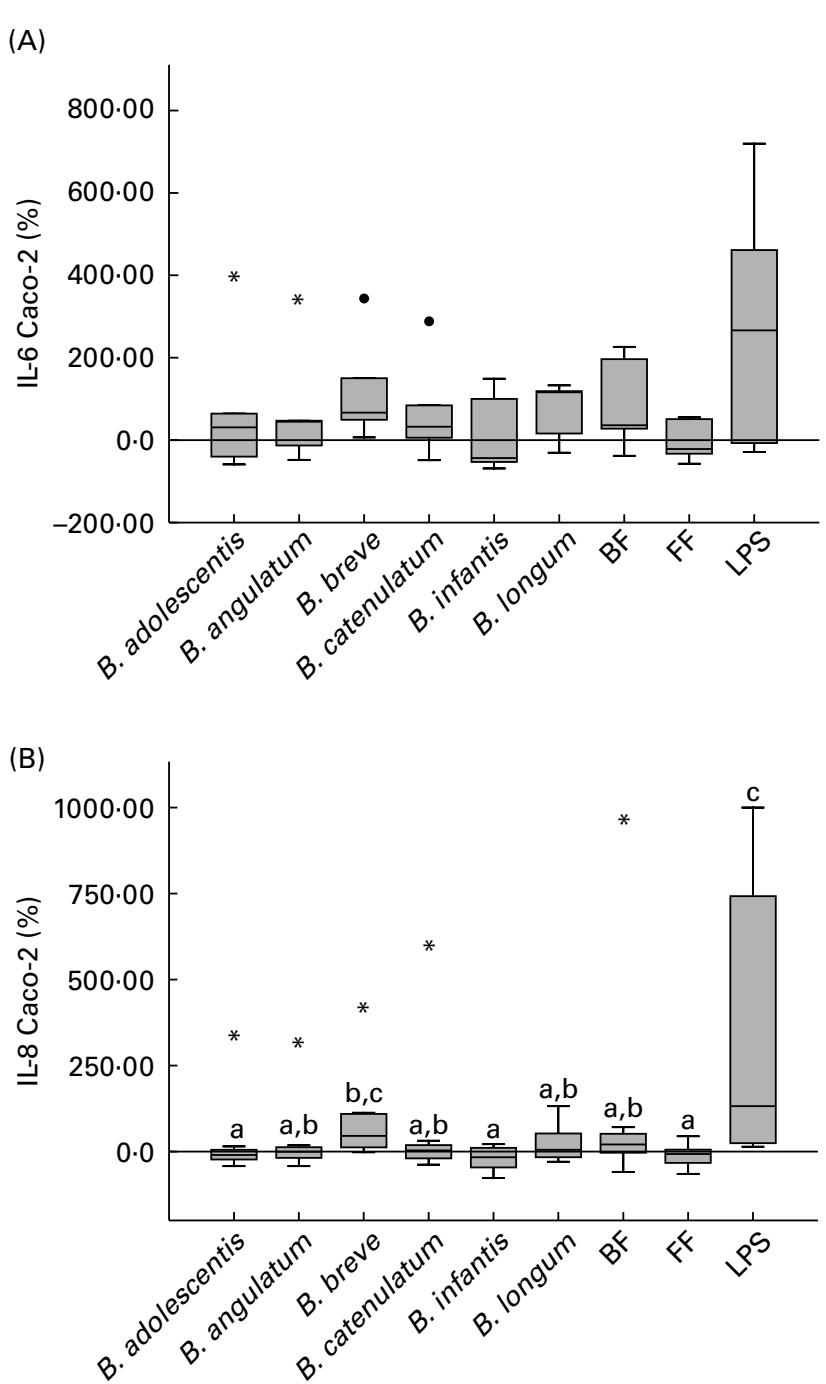

Fig. 3. Bifidobacteria-stimulated cytokine production by Caco-2 cells in a $36 \mathrm{~h}$ culture (basolateral medium) following prior $12 \mathrm{~h}$ sensitisation with peripheral blood mononuclear cells in a transwell co-culture system. Values are given as percentage of the control (spontaneous production with no added bacteria). Each box represents median (50th percentile) and interquartile range (25th and 75th percentiles). Asterisks and dots represent outliers and extreme values, respectively. ${ }^{\mathrm{a}, \mathrm{b}, \mathrm{c}}$ Mean values with unlike letters were significantly different $(P<0.05$; Mann-Whitney $U$ test). No differences were observed in IL-6 production between the conditions. (A) IL-6 Caco-2; (B) IL-8 Caco-2. BF, breast fed; FF, formula fed; LPS, lipopolysaccharide.

tolerance and balanced intestinal immune responses in the early stages of life. Both these processes have been linked to the prevention of immune-mediated disorders later in life, such as allergies or inflammatory bowel disease ${ }^{(17,18)}$. Several studies have evaluated the effect of different bifidobacteria in the production of cytokines by Caco-2 cells and PBMC $^{(6,9,19-21)}$, but according to our knowledge, this is the first time that the Bifidobacterium strains used in the present study have been employed in co-culture experiments, and that the mixtures in the proportions of a FF and BF infant's typical microbiota have been used to stimulate these cell types.

In the present study, the levels of several cytokines were measured in two different systems: (1) a direct stimulation of PBMC with bifidobacteria and (2) a PBMC/Caco-2 cell 
co-culture with bifidobacteria stimulating the top layer of Caco-2 cells, which, in turn, can interact with underlying PBMC through soluble mediators. Reciprocally, PBMC were able to influence Caco-2 cell activity as well. The profile of cytokine production by PBMC exposed directly to the Bifidobacterium strains shows relevant differences compared with the profile of cytokine production by PBMC in the co-culture system, where Caco-2 cells constitute a physical barrier preventing the access of PBMC to the bifidobacteria. The first differential finding was that the level of cytokine production was much lower in the co-culture system. For instance, while three out of six cytokines measured were above $1000 \mathrm{pg} / \mathrm{ml}$ when both bifidobacterial mixtures were used, and two out of the remaining three gave results higher than $100 \mathrm{pg} / \mathrm{ml}$ in direct contact, only IL- 6 by PBMC in the co-culture system gave results higher than $1000 \mathrm{pg} / \mathrm{ml}$. It is worth noting that while in direct contact, IL- 6 and TNF- $\alpha$ were the cytokines most highly induced, in the co-culture system, not only IL-6 but also IL-10 was the cytokine most highly produced by PBMC. In this sense, Niers et al. showed in a single culture system that the production of IL-10 by PBMC is boosted by several Bifidobacterium strains, and this down-regulates the production of TNF- $\alpha$ and IL-12p70 by these cells. When they used a monoclonal antibody against IL-10, they found a huge increase in the production of these inflammatory cytokines

Different cytokines (IL-8 and IL-6) were also stimulated on Caco-2 cells, but only when they were previously co-cultured with PBMC; no cytokine production was measured if the Caco-2 cells were cultured alone with the Bifidobacterium strains. Therefore, the presence of PBMC is an essential factor for the sensitisation of Caco-2 cells to respond to bifidobacteria, which is presumably exerted by the communication between the two cell types through soluble mediators. In the present study and other studies, Caco-2 cells alone have been found to be hyporesponsive to bifidobacteria stimulation $^{(22)}$ and also to other probiotic bacteria ${ }^{(21,22)}$. Moreover, since cytokine production by $\mathrm{CacO}-2$ cells in the co-culture system was only detectable in the basolateral medium and not in the apical medium, it demonstrates a polarised secretion by Caco-2 cells, as have been found earlier by other authors $^{(21)}$. In a similar co-culture system, in which Caco-2 cells were stimulated with non-pathogenic Escherichia coli and Lactobacillus sakei, an induction of TNF- $\alpha$ secretion into the subepithelial compartment was observed, and this cytokine was signalled as the fundamental candidate for cellular crosstalk $^{(21)}$. In contrast, we found no detectable production of TNF- $\alpha$, which might be explained by a differential effect from different bacterial species and strains.

Regarding the immunomodulatory effects of specific strains used in these experiments, the most relevant findings have been found regarding the immunostimulatory effects of $B$. breve. This strain stimulated most of the production of IL- 8 and IL- 6 on both Caco- 2 cells and PBMC. In the microbiota of $\mathrm{BF}$ infants, $B$. breve is the most representative Bifidobacterium species (after B. infantis, common in all milk-fed babies), and this could explain the high IL- 8 and IL- 6 levels produced by $\mathrm{CacO}-2$ and $\mathrm{PBMC}$ stimulated with the $\mathrm{BF}$ mixture. This link between $B$. breve and the $\mathrm{BF}$ mixture was supported by the correlation found between IL-8 levels produced by Caco- 2 cells stimulated by $B$. breve and the BF mixture. Moreover, $B$. breve and the $\mathrm{BF}$ mixture also stimulated the production of IL-10 and INF- $\gamma$ by PBMC (in co-culture with Caco-2 cells). All these observations might indicate that the proportion of different Bifidobacterium species is an important determinant of the overall contribution to the stimulation of cytokines on the intestinal mucosa. In this sense, it is interesting to note that there was a correlation between the relative inhibition of IL- 8 production by Caco- 2 cells induced by the $\mathrm{FF}$ mixture and $B$. infantis. It seems that the differences in the proportions of the different strains between the mixtures and the stimulatory/inhibitory capacities shown by the individual strains might explain the results found with their combinations in the $\mathrm{BF}$ and $\mathrm{FF}$ mixtures.

According to the results, $B$. breve induced a slight proinflammatory response, which could turn the mucosal immune system on stand-by and prevent the release of a severe inflammation. It has already been reported that infants from 4 to 6 months old, who daily consumed infant formula fermented with B. breve and Streptococcus thermophilus, presented less severe episodes of acute diarrhoea than the standard formula group ${ }^{(23)}$. Furthermore, Li et al. $^{(24)}$ showed that the administration of $B$. breve to low-birth-weight infants was useful in promoting the colonisation by other bifidobacteria, which might contribute to the establishment of a healthier microbiota. More recently, it has been found that the administration of $B$. breve to pre-term infants can up-regulate transforming growth factor- $\beta 1$ signalling and may possibly be beneficial in attenuating inflammatory and allergic reactions in these infants ${ }^{(25)}$.

In allergic models, some probiotic bifidobacteria have the capacity to suppress IL-4 production, in vitro ${ }^{(16)}$ and in vivo ${ }^{(26)}$. We have observed that not all bifidobacterial species induce the same IL-4 production (Fig. 1(D)), indicating different effects of the interaction between bifidobacteria and PBMC related to the species.

Regarding the stimulation of the regulatory cytokine IL-10 by PBMC after direct stimulation with B. longum, a similar finding has been previously described by Medina et al., who found that several strains of B. longum are strong inducers of IL-10 secretion on PBMC. On the other hand, the finding that $B$. infantis is a weak inducer of cytokine secretion after direct stimulation of both PBMC and Caco-2 cells is in agreement with prior published results that have described that B. infantis attenuates baseline IL-8 secretion in HT-29 epithelial cells ${ }^{(5)}$ as well as pro-inflammatory IL-17 production by murine splenocytes and dextran sodium sulphate-induced intestinal inflammation ${ }^{(27,28)}$.

In conclusion, among the Bifidobacterium species tested, $B$. breve seems to be the most immunostimulatory strain in a co-culture system resembling the physiological layout of different cell types in the intestinal mucosa. The presence and relative proportions of different Bifidobacterium species in the microbiota of $\mathrm{BF}$ and $\mathrm{FF}$ infants could be key factors defining the immunomodulatory effect of the gut microbiota in early life. 


\section{Acknowledgements}

The present study was supported by grants AGL2007-66126C03-01/ALI and AGL2007-66126-C03-03/ALI, from the Spanish Ministry of Science and Innovation, and grants 200570F0091, 200570 F0093 and $200870 I 183$ from the CSIC. T. P.-R. was the recipient of a personal grant from the JAE/I3P Program of CSIC (Spain). The authors have no conflicts of interest in the present study. E. N., A. M. and Y. S. designed the study; T. P.-R., E. P. and E. N. conducted most of the study; I. N. performed all the microbiological experiments; T. P.-R., J. R. M. and E. N. contributed equally to the discussion and to the writing of the manuscript; Y. S. and A. M. reviewed and contributed to the writing of the final version of the manuscript.

\section{References}

1. Candela M, Perna F, Carnevali P, et al. (2008) Interaction of probiotic Lactobacillus and Bifidobacterium strains with human intestinal epithelial cells: adhesion properties, competition against enteropathogens and modulation of IL-8 production. Int J Food Microbiol 125, 286-292.

2. Duerkop BA, Vaishnava S \& Hooper LV (2009) Immune responses to the microbiota at the intestinal mucosal surface. Immunity 31, 368-376.

3. Honda K \& Takeda K (2009) Regulatory mechanisms of immune responses to intestinal bacteria. Mucosal Immunol 2, 187-196.

4. Zeuthen LH, Fink LN \& Frokiaer H (2008) Epithelial cells prime the immune response to an array of gut-derived commensals towards a tolerogenic phenotype through distinct actions of thymic stromal lymphopoietin and transforming growth factor-beta. Immunology 123, 197-208.

5. O'Hara AM, O'Regan P \& Fanning A (2006) Functional modulation of human intestinal epithelial cell responses by Bifidobacterium infantis and Lactobacillus salivarius. Immunology 118, 202-215.

6. Parlesak A, Haller D \& Brinz S (2004) Modulation of cytokine release by differentiated CACO-2 cells in a compartmentalized coculture model with mononuclear leucocytes and nonpathogenic bacteria. Scand J Immunol 60, $477-485$.

7. He F, Ouwehand AC, Isolauri E, et al. (2001) Comparison of mucosal adhesion and species identification of bifidobacteria isolated from healthy and allergic infants. FEMS Immunol Med Microbiol 30, 43-47.

8. Latvala S, Pietila TE, Veckman V, et al. (2008) Potentially probiotic bacteria induce efficient maturation but differential cytokine production in human monocyte-derived dendritic cells. World J Gastroenterol 14, 5570-5583, discussion $81-2$.

9. Niers LE, Timmerman HM, Rijkers GT, et al. (2005) Identification of strong interleukin-10 inducing lactic acid bacteria which down-regulate $\mathrm{T}$ helper type 2 cytokines. Clin Exp Allergy 35, 1481-1489.

10. Haarman M \& Knol J (2005) Quantitative real-time PCR assays to identify and quantify fecal Bifidobacterium species in infants receiving a prebiotic infant formula. Appl Environ Microbiol 71, 2318-2324.

11. Mountzouris KC, McCartney AL \& Gibson GR (2002) Intestinal microflora of human infants and current trends for its nutritional modulation. Br J Nutr 87, 405-420.
12. Furrie E, Macfarlane S, Kennedy A, et al. (2005) Synbiotic therapy (Bifidobacterium longum/Synergy 1) initiates resolution of inflammation in patients with active ulcerative colitis: a randomised controlled pilot trial. Gut 54, 242-249.

13. Kalliomaki M, Kirjavainen P, Eerola E, et al. (2001) Distinct patterns of neonatal gut microflora in infants in whom atopy was and was not developing. J Allergy Clin Immunol 107, $129-134$.

14. Sanz Y, Sánchez E, De Palma G, et al. (2008) Indigenous gut microbiota, probiotics, and coeliac disease. In Child Nutrition and Physiology, pp. 211-224 [LT Overton and MR Ewente, editors]. New York: Nova Science Publishers, Inc.

15. Hua MC, Lin TY, Lai MW, et al. (2010) Probiotic bio-three induces Th1 and anti-inflammatory effects in PBMC and dendritic cells. World J Gastroenterol 16, 3529-3540.

16. Ghadimi D, Fölster-Holst R, de Vrese M, et al. (2008) Effects of probiotic bacteria and their genomic DNA on TH1/TH2cytokine production by peripheral blood mononuclear cells (PBMCs) of healthy and allergic subjects. Immunobiology 213, 677-692.

17. Kelly D, King $\mathrm{T} \&$ Aminov R (2007) Importance of microbial colonization of the gut in early life to the development of immunity. Mutat Res 622, 58-69.

18. Conroy ME, Shi HN \& Walker WA (2009) The long-term health effects of neonatal microbial flora. Curr Opin Allergy Clin Immunol 9, 197-201.

19. Medina M, Izquierdo E, Ennahar S, et al. (2007) Differential immunomodulatory properties of Bifidobacterium logum strains: relevance to probiotic selection and clinical applications. Clin Exp Immunol 150, 531-538.

20. Haller D, Holt L, Parlesak A, et al. (2004) Differential effect of immune cells on non-pathogenic Gram-negative bacteria-induced nuclear factor-kappaB activation and pro-inflammatory gene expression in intestinal epithelial cells. Immunology 112, 310-320.

21. Haller D, Bode C, Hammes WP, et al. (2000) Non-pathogenic bacteria elicit a differential cytokine response by intestinal epithelial cell/leucocyte co-cultures. Gut 47, 79-87.

22. Morita H, He F, Fuse T, et al. (2002) Adhesion of lactic acid bacteria to caco-2 cells and their effect on cytokine secretion. Microbiol Immunol 46, 293-297.

23. Thibault H, Aubert-Jacquin C \& Goulet O (2004) Effects of long-term consumption of a fermented infant formula (with Bifidobacterium breve c50 and Streptococcus thermophilus 065) on acute diarrhea in healthy infants. $J$ Pediatr Gastroenterol Nutr 39, 147-152.

24. Li Y, Shimizu T, Hosaka A, et al. (2004) Effects of Bifidobacterium breve supplementation on intestinal flora of low birth weight infants. Pediatr Int 46, 509-515.

25. Fujii T, Ohtsuka Y, Lee T, et al. (2006) Bifidobacterium breve enhances transforming growth factor beta1 signaling by regulating Smad7 expression in preterm infants. $J$ Pediatr Gastroenterol Nutr 43, 83-88.

26. Zhang LL, Chen X, Zheng PY, et al. (2010) Oral Bifidobacterium modulates intestinal immune inflammation in mice with food allergy. J Gastroenterol Hepatol 25, 928-934.

27. Osman N, Adawi D, Molin G, et al. (2006) Bifidobacterium infantis strains with and without a combination of oligofructose and inulin (OFI) attenuate inflammation in DSS-induced colitis in rats. BMC Gastroenterol 6, 31.

28. Tanabe S, Kinuta Y \& Saito Y (2008) Bifidobacterium infantis suppresses proinflammatory interleukin-17 production in murine splenocytes and dextran sodium sulfate-induced intestinal inflammation. Int J Mol Med 22, 181-185. 